
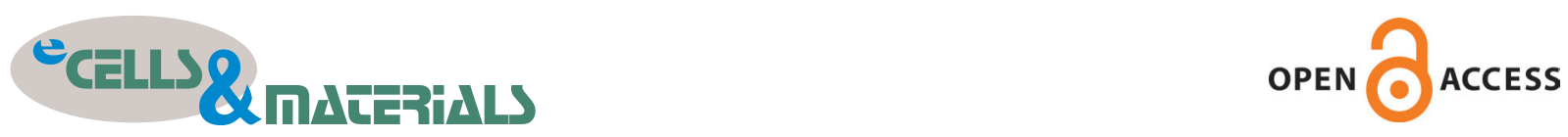

\title{
ANIMAL MODELS FOR STUDYING METAPHYSEAL BONE FRACTURE HEALING
}

\author{
M. Haffner-Luntzer ${ }^{1, *}$ and A. Ignatius ${ }^{1}$ \\ ${ }^{1}$ Institute of Orthopaedic Research and Biomechanics, University Medical Centre Ulm, Germany
}

\begin{abstract}
An estimated 2 million osteoporotic fractures occur annually in the US, resulting in a dramatic reduction in quality of life for affected patients and a high economic burden for society. Osteoporotic fractures are frequently located in metaphyseal bone regions. They are often associated with healing complications, because of the reduced healing capacity of the diseased bone tissue, the poor primary stability of the fracture fixation in the fragile bone, and the high frequency of comorbidities in these patients. Therefore, osteoporotic fractures require optimised treatment strategies to ensure proper bone healing. Preclinical animal models can help understanding of the underlying mechanisms and development of new therapies. However, whereas diaphyseal fracture models are widely available, appropriate animal models for metaphyseal fracture healing are scarce, although essential for translational research. This review covers large and small animal models for metaphyseal fracture healing. General requirements for suitable animal models are presented, as well as advantages and disadvantages of the current models. Furthermore, differences and similarities between metaphyseal and diaphyseal bone fracture healing are discussed. Both large- and small-animal models are available for studying metaphyseal fracture healing, which mainly differ in fracture location and geometry as well as stabilisation techniques. Most common used fracture sites are distal femur and proximal tibia. Each model found in the literature has certain advantages and disadvantages; however, many lack standardisation resulting in a high variability or poor mimicking of the clinical situation. Therefore, further refinement of animal models is needed especially to study osteoporotic metaphyseal fracture healing.
\end{abstract}

Keywords: Fracture healing, animal models, metaphyseal fractures, bone regeneration.

*Address for correspondence: Dr Melanie Haffner-Luntzer, Helmholtzstraße 14, 89081 Ulm, Germany. Telephone number: + 4973150055301 Email: melanie.haffner-luntzer@uni-ulm.de

Copyright policy: This article is distributed in accordance with Creative Commons Attribution Licence (http://creativecommons.org/licenses/by-sa/4.0/).

\section{Introduction}

Long bones are anatomically divided into the metaphyseal areas at each end and the diaphyseal region in between the metaphyseal areas. Metaphyseal bone is rich in trabecular bone surrounded by a thin cortical shell. In contrast, diaphyseal bone consist of a dense cortical bone shell enclosing the marrow cavity. Trabecular bone is more metabolically active compared to cortical bone due to its larger surface, the higher number of osteoblasts and osteoclasts as well as a good blood supply (Claes et al., 2011b; Fan et al., 2008). It was previously shown that osteoporosis, a disease characterised by bone loss and microarchitectural deterioration, affects trabecular bone rather than cortical bone at the beginning of the disease (Peng et al., 1994). Therefore, many fractures in osteoporotic patients occur in metaphyseal bone. Most common fracture sites are the distal radius, proximal humerus, proximal femur and the spine. Each year an estimated 2 million osteoporotic fractures happen in the US, resulting in a dramatic reduction in quality of life for affected patients and a great economic burden for society (Alt and Miclau, 2016). Worldwide, one out of three women and one out of five men aged 50 years and over are at risk of osteoporotic fractures. Healing of osteoporotic fractures is often delayed due to increased osteoclast activity, decreased endochondral and intramembranous bone formation in the fracture callus, reduced angiogenesis and a disturbed inflammatory response towards fracture (Beil et al., 2010; Haffner-Luntzer et al., 2017; Nikolaou et al., 2009). Besides this reduced healing capacity of the diseased osteoporotic bone tissue, osteoporotic fractures are challenging to treat because the weak bone quality hampers stable fracture fixation resulting in unfavourable mechanical conditions. Therefore, optimised fracture treatment strategies are required to reduce the increased complication rate in osteoporotic patients (Nikolaou et al., 2009). 
Preclinical animal models are essential for the development of new implants and therapeutics. So far, most animal models have recapitulated diaphyseal fracture healing. However, clinical observations and preclinical studies have indicated that diaphyseal and metaphyseal fracture healing may differ in many aspects, including the time course of healing, the extent of callus formation, the proportion of intramembranous and endochondral ossification, the source of precursor cells, the role of inflammation in the healing process, and the influence of the mechanical environment on the healing process (Sandberg and Aspenberg, 2016; Tatting et al., 2018). Thus, the knowledge gained from diaphyseal healing models could not be simply transferred to metaphyseal healing. To better understand the characteristics of metaphyseal bone healing in general, and to unravel the pathomechanisms of disturbed metaphyseal healing observed in osteoporotic patients, suitable preclinical models are needed. They are a prerequisite for the development of new treatment strategies.

Here, an overview is provided of the current animal models of metaphyseal fracture healing. The advantages and limitations of the models and possible differences between metaphyseal and diaphyseal fracture healing are discussed.

\section{Requirements for animal models to study metaphyseal fracture healing}

The selection of a suitable animal model is of utmost importance to answer specific research questions and to transfer the results to the clinical situation. A preclinical model should ideally closely mimic the human situation. Differences and similarities between the model organism and the human species must be considered in general and specifically with respect to the experimentally induced fracture and the concomitant disorder (e.g. osteoporosis). It is essential that the metaphyseal fracture model is highly standardised and reproducible to draw valid conclusions from the experiments. Technical variation should be eliminated or substantially reduced. Further, animal welfare, as well as time and cost issues play a role in the selection of the animal model.

Large animals, such as sheep and dogs, have been used for decades in fracture healing research, and some of these studies also addressed metaphyseal bone healing (Fig. 1). Compared to small animals, large animals more closely mimic human bone physiology in terms of bone metabolism, dimensions, and mechanical loading conditions. Often, clinically available fracture fixation devices can be used due to the comparable skeletal size. Furthermore, fracture healing can be evaluated using similar tools

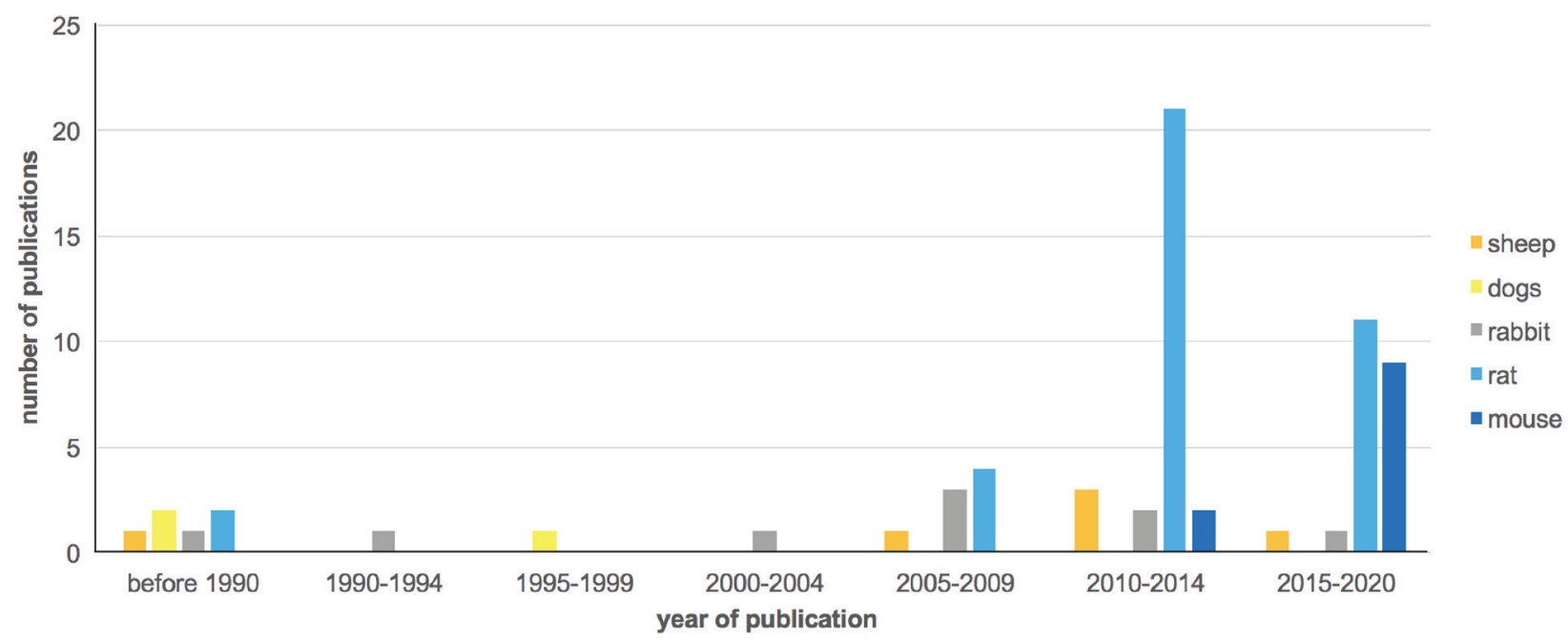

Fig. 1. Usage of different animal species for metaphyseal fracture healing research over time. Number of publications found in PMC by a systematic literature research which was performed in early June 2020. The first search was performed using the search expression "metaphyseal" AND "fracture". This search resulted in 1963 publications. The second search was performed using the search expression "cancellous" AND "fracture" NOT "metaphyseal". This search resulted in 3190 publications. The third search strategy was performed using the search expression "osteotomy" AND "metaphyseal" NOT "fracture". This search resulted in 242 publications. In total 5395 publications were screened. 66 publications were selected for this review based on certain inclusion and exclusion criteria. Inclusion criteria were: preclinical study, in vivo study, fracture model, metaphyseal bone involved, full-text available in English, German or French. Exclusion criteria were: clinical study, case study from veterinarian medicine, ex vivo or in vitro study, no fracture model (e.g. osteolysis or bone defect model without implant fixation), no metaphyseal bone involved, published as conference abstracts or full text not available. Within the selected 66 publications, there were 5 sheep studies, 3 dog studies, 9 rabbit studies, 38 rat studies and 11 mouse studies. Simple bone drill-hole models were not included in this literature review, because they reflect defect healing rather than metaphyseal fracture healing and lack adequate mechanical stimulation. 
to those used in the clinics, such as radiography and CT imaging. Additionally, as a benefit of animal models in general, ex vivo analysis such as histological evaluation is possible. On the other hand, large animal experiments are space and cost intensive and possible molecular analyses are limited, because transgenic animals are hardly available and molecular analytical tools (e.g. antibodies) often missing (Oheim et al., 2016). Furthermore, the usage of drugs in the experimental setting can be expensive due to the large body weight. Importantly, the induction of osteoporosis to study osteoporotic fracture healing is challenging in sheep (Oheim et al., 2016). Ovariectomy (OVX), a commonly used model to mimic postmenopausal osteoporosis, is not sufficient to induce significant bone loss in sheep. OVX needs to be combined with other treatments such as glucocorticoid application, calcium deficient diet or surgical procedures, e.g. disconnection of the hypothalamus-pituitary axis (Oheim et al., 2013; Oheim et al., 2016; Oheim et al., 2017), making these models much more complex and challenging their translational relevance.

In recent years small animals, including rabbits, rats and mice, have become more and more popular for metaphyseal fracture healing research (Fig. 1). These species are relatively easy to handle, as well as being cost and space effective. Further, the bone healing process is much faster in small animals compared to larger species, resulting in shorter experimental time periods. A major advantage is the availability of genetically modified animals, which allows the unravelling of molecular mechanisms of the fracture healing process. This is especially true for mice, but there are also some transgenic rabbit and rat lines available. Furthermore, the small size of the animals allows cost-effective treatments with drugs, antibodies, or other biological substances of interest. Additionally, significant bone loss can be induced in rodents by OVX and aging, if a study of metaphyseal fracture healing in a postmenopausal model or agerelated osteoporosis is desired. The disadvantage of small animal models is that bone structure, metabolism and size are very different from humans. Furthermore, the loading situation is not comparable. Due to the small skeletal size, technical problems can arise in creating standardised fractures. Custom fracture fixation devices need to be developed as well as standardised surgical techniques. Since the metaphyseal area in the long bones of adult mice is very small, it can be challenging to establish a fracture protocol especially for those animals.

Basically, standardised mechanical conditions at the fracture site are essential to reduce interindividual variation and gain reproducible data, because bone healing is highly dependent on the tissue strains acting at the fracture site (Claes, 2017). Small interfragmentary strains may result in intramembranous ossification, the direct conversion of mesenchymal tissue into bone, whereas moderate strains induce endochondral ossification, in which a cartilaginous matrix intermediate is converted to bone (also known as callus healing). High interfragmentary strains impair bone healing. This seems to be true for both diaphyseal and metaphyseal fracture healing (Claes et al., 2011a; Hinsenkamp et al., 1984; Sandberg and Aspenberg, 2016; Uhthoff and Rahn, 1981). The mechanical conditions in the healing zone are determined by the location and geometry of the fracture, the stability of fracture fixation and the mechanical loading of the fractured limb. Therefore, the model must be highly standardised with respect to these features. Furthermore, from a translational perspective a suitable animal model should closely mimic fracture types observed in the clinics.

\section{Methodology for systematic literature research}

A systematic literature research was performed using PubMed Central ${ }^{\circledR}$ (PMC) in early June 2020.

The first search was performed using the search expression "metaphyseal" AND "fracture". This search resulted in 1963 publications. The second search was performed using the search expression "cancellous" AND "fracture" NOT "metaphyseal". This search resulted in 3190 publications. The third search strategy was performed using the expression "osteotomy" AND "metaphyseal" NOT "fracture". This search resulted in 242 publications.

In total, 5395 publications were screened. 66 publications were selected for this review, based on certain inclusion and exclusion criteria. Inclusion criteria were: preclinical study, in vivo study, fracture model, metaphyseal bone involved, full-text available in English, German or French. Exclusion criteria were: clinical study, case study from veterinarian medicine, ex vivo or in vitro study, no fracture model (e.g. osteolysis or bone defect model without implant fixation), no metaphyseal bone involved, published as conference abstracts or full text not available.

Within the selected 66 publications, there were 5 sheep studies, 3 dog studies, 9 rabbit studies, 38 rat studies and 11 mouse studies. Simple bone drillhole models were not included in this literature review, because they reflect defect healing rather than metaphyseal fracture healing and lack adequate mechanical stimulation.

\section{Large animal models for metaphyseal fracture healing}

\section{Sheep}

Studies using sheep as a model organism for metaphyseal fracture healing research are scarce. Only 5 papers were found in PMC. The studies differ in the localisation of the fracture and the possibility of defining and controlling the mechanical conditions at the fracture site (Table 1).

Heiss et al. developed a metaphyseal fracture model at the proximal ulna with the primary aim 
of testing a new bone glue (Heiss et al., 2010). A metaphyseal segment of $20 \mathrm{~mm}$ length was removed from the proximal ulna, reinserted, and then fixed with the bone adhesive or not (control animals). The authors reported that the sheep loaded their leg normally from the first postoperative day onwards, indicating that the procedure was well tolerated. Most fractures were bridged with newly formed bone after 3 months. External callus formation was only observed in the control group, suggesting that the fracture was unstable, if the fragment was not fixed with the glue. The advantages of this metaphyseal fracture model were early weight-bearing, even if the bone segment was not fixed, and - with respect to animal welfare - the absence of adverse events in the postoperative period. However, the results showed a relatively high variance within the groups, suggesting difficulties in technical standardisation and/or varying mechanical conditions. Furthermore, the location of the fracture at the proximal ulna does not reflect a common clinical situation.

The other available studies used metaphyseal fracture models at the distal femur (Alagboso et al., 2019; Bindl et al., 2013; Claes et al., 2011a; Claes et al., 2009). In one study, a full wedge-shaped osteotomy was created at the lateral femoral condyle, which was rigidly stabilised by a custom-made locking plate (Alagboso et al., 2019). All animals tolerated the surgery well and returned to full weight-bearing within the first 3 postoperative days. After 3 months, intramembranous bone formation was observed in the fracture gap with some variation in bony bridging scores (healing scores ranged from 4 to 9) within the groups. Biomechanical indentation testing revealed no significant differences in stiffness between healed and intact bone indicating successful healing. No external callus was found at the lateral aspect of the osteotomy, only a small periosteal callus was observed at its medial site. In comparison to the abovementioned model, this sheep model might provide a higher degree of standardisation. Furthermore, the authors state that the full discontinuity of the osteotomy gap may better reflect the clinical situation than a partial osteotomy at the distal femur as used in other studies (Claes et al., 2011a; Claes et al., 2009).

In order to study the influence of mechanical loading on metaphyseal fracture healing, Claes et al. also established a sheep model at the distal femur (Claes et al., 2009). A partial osteotomy ( $3 \mathrm{~mm}$ ) was created at the femoral condyle close to the trochlea. The deflection of the osteotomy gap during limb loading was controlled by distance plates inserted at the proximal end of the gap. All animals tolerated the surgery well and returned to normal activity after $3 \mathrm{~d}$. Bone healing was analysed after 8 weeks in different regions of interest in the osteotomy gap that displayed different magnitudes of interfragmentary strains. In areas with strains below $5 \%$, significantly less bone was formed compared to areas with higher strains (6 to $20 \%$ ). Strains larger than $20 \%$ induced fibrocartilage formation. From these results, it was suggested that metaphyseal bone healing follows similar biomechanical principles to diaphyseal healing, although no external callus was formed. This metaphyseal fracture model was also used in an osteoporotic sheep model (Bindl et al., 2013). The advantages of this model are the high degree of standardisation and the exactly controllable mechanical conditions.

In summary, to date only few models have been established for studying metaphyseal fracture healing in sheep. Most studies have been conducted

Table 1. Sheep models for metaphyseal fracture healing.

\begin{tabular}{|c|c|c|c|c|c|c|c|}
\hline Strain & Age & Sex & $\begin{array}{l}\text { Fracture } \\
\text { site }\end{array}$ & Fracture & $\begin{array}{c}\text { Osteoporosis } \\
\text { model }\end{array}$ & Healing outcome & References \\
\hline $\begin{array}{c}\text { mountain } \\
\text { sheep, } \\
\text { black } \\
\text { head } \\
\text { sheep, } \\
\text { merino } \\
\text { sheep }\end{array}$ & $\begin{array}{c}3-6 \\
\text { years }\end{array}$ & female & $\begin{array}{l}\text { distal } \\
\text { femur }\end{array}$ & $\begin{array}{c}\text { open partial } \\
\text { osteotomy } \\
(3 \mathrm{~mm} \text { gap }), \\
\text { plate implanted } \\
\text { to limit } \\
\text { interfragmentary } \\
\text { movement }\end{array}$ & $\begin{array}{l}\text { ovariectomy, } \\
\text { hypothalamic- } \\
\text { pituitary } \\
\text { disconnection }\end{array}$ & $\begin{array}{l}\text { interfragmentary } \\
\text { strains at the } \\
\text { fracture site } \\
\text { determine type of } \\
\text { tissue formation: } \\
\text { endochondral and } \\
\text { intramembranous } \\
\text { bone formation }\end{array}$ & $\begin{array}{l}\text { Claes et al., } \\
2009 \\
\text { Claes et al., } \\
2010 \\
\text { Bindl et al., } \\
2013\end{array}$ \\
\hline $\begin{array}{l}\text { merino } \\
\text { sheep }\end{array}$ & $\begin{array}{c}5-8 \\
\text { years }\end{array}$ & female & $\begin{array}{l}\text { proximal } \\
\text { ulna }\end{array}$ & $\begin{array}{l}\text { open osteotomy } \\
\text { ( } 20 \mathrm{~mm} \text { gap), no } \\
\text { fixation or bone } \\
\text { glue fixation }\end{array}$ & N/A & $\begin{array}{c}\text { healing by } \\
\text { intramembranous } \\
\text { bone formation }\end{array}$ & $\begin{array}{l}\text { Heiss et al., } \\
2010\end{array}$ \\
\hline $\begin{array}{l}\text { merino } \\
\text { sheep }\end{array}$ & $\begin{array}{l}6-7 \\
\text { years }\end{array}$ & female & $\begin{array}{l}\text { distal } \\
\text { femur }\end{array}$ & $\begin{array}{l}\text { open wedge- } \\
\text { shaped } \\
\text { osteotomy, plate } \\
\text { fixation }\end{array}$ & N/A & $\begin{array}{l}\text { healing by } \\
\text { intramembranous } \\
\text { bone formation }\end{array}$ & $\begin{array}{c}\text { Alagboso et al., } \\
2019\end{array}$ \\
\hline
\end{tabular}


at the distal femur. Depending on the localisation and stabilisation of the fractures, healing occurs in 2 to 3 months without pronounced external callus formation. To our best knowledge, only one study used osteoporotic sheep to mimic metaphyseal fracture healing in osteoporotic patients.

\section{Dogs}

Only 3 studies were found which used dogs to study metaphyseal fracture healing, all of them at the distal femur (Table 2). Uhthoff et al. created a standardised wedge-shaped fracture gap in the distal femur (Uhthoff and Rahn, 1981). Since the osteotomy was incomplete, no fixation device was used. After 4 weeks, the fractures displayed the first signs of intramembranous ossification, suggesting a high mechanical stability. Later time points were not investigated. Furthermore, the same group established an intercondylar femoral osteotomy model (Uhthoff et al., 1987). A lateral osteotomy was sawed, the bone fragments reduced and fixed by two cortical screws. The authors reported that the operation was well tolerated and that all fractures healed uneventfully by direct bone formation within 14 weeks. This was confirmed by another group that used the same model to test bioabsorbable screws (An et al., 1998). This model is highly standardised due to the standardised osteotomy and the complete reduction of the fracture gap.

In conclusion, the described models resulted in direct bone formation at the fracture site. Complete healing took up to 14 weeks. Ethical considerations might be the reason for the low number of studies in dogs. No osteoporotic dog models were used.

\section{Small animal models for metaphyseal fracture healing}

\section{Rabbits}

Rabbits have been used for a long time as a model organism for metaphyseal fracture healing research (Table 3). Fracture locations vary from proximal fibula, proximal and distal tibia and distal femur. In fact, rabbits are the model organism with the widest spectrum of described models at different fracture sites.

Matos et al. established an osteotomy model in the proximal rabbit fibula, which was stabilised by the intact tibia (Matos et al., 2007). The very stable and standardised fracture geometry led to predominantly intramembranous bone formation. Furthermore, the authors were able to demonstrate a robust osteoanabolic effect of zoledronic acid in this model. However, the proximal fibula is not a common location for metaphyseal fractures in the clinics.

Tsiridis et al. (Tsiridis et al., 2007) created a unicortical, wedge-shaped osteotomy at the distal tibia. The lateral gap size was $4 \mathrm{~mm}$. The fracture was fixed by a custom-made external fixator. All fractures were healed after 4 weeks and did not display external callus formation. Advantages of this model are the clinically relevant fracture location and the good reproducibility. This model was successfully

Table 2. Dog models for metaphyseal fracture healing.

\begin{tabular}{|c|c|c|c|c|c|c|c|}
\hline Strain & Age & Sex & $\begin{array}{c}\text { Fracture } \\
\text { site }\end{array}$ & Fracture mo & $\begin{array}{c}\text { Osteoporosis } \\
\text { model }\end{array}$ & Healing outcome & References \\
\hline Beagles & adult & N/A & $\begin{array}{l}\text { distal } \\
\text { femur }\end{array}$ & $\begin{array}{l}\text { incomplete osteotomy, } \\
\text { no fixation }\end{array}$ & N/A & $\begin{array}{l}\text { appearance of external } \\
\text { callus depends on } \\
\text { fracture stability }\end{array}$ & $\begin{array}{l}\text { Uhthoff et } \\
\text { al., } 1981\end{array}$ \\
\hline N/A & $\begin{array}{c}3-8 \\
\text { years }\end{array}$ & N/A & $\begin{array}{l}\text { distal } \\
\text { femur }\end{array}$ & $\begin{array}{c}\text { open intercondylar } \\
25 \text { mm long osteotomy, } \\
\text { fixation with cortical } \\
\text { screws }\end{array}$ & N/A & $\begin{array}{l}\text { healing by direct bone } \\
\text { formation }\end{array}$ & $\begin{array}{l}\text { Uhthoff et } \\
\text { al., } 1987\end{array}$ \\
\hline N/A & adult & male & $\begin{array}{l}\text { distal } \\
\text { femur }\end{array}$ & $\begin{array}{c}\text { open lateral femoral } \\
\text { condyle osteotomy, } \\
\text { fixation with two } \\
\text { screws }\end{array}$ & N/A & $\begin{array}{l}\text { healing by direct bone } \\
\text { formation }\end{array}$ & $\begin{array}{l}\text { An et al., } \\
1998\end{array}$ \\
\hline
\end{tabular}


used to analyse the effects of parathyroid hormone (PTH) and bone morphogenetic protein (BMP)-7 on metaphyseal fracture healing (Morgan et al., 2008; Tsiridis et al., 2007).

Other models were created at the proximal tibia. Han et al. introduced an oblique proximal tibia fracture model (Han et al., 2012). They created a bilateral splitting fracture through the tibial plateau. The fracture was fixed by a lateral compression screw. The authors reported that only one animal suffered from fracture displacement, indicating a high degree of standardisation in this model. No external callus was formed, and histological analysis revealed osteoblast proliferation along the bone trabeculae as early as 1 week after fracture and intramembranous bone formation after 2 weeks. Bony bridging occurred 4 weeks after surgery. Chen et al. published a vertical osteotomy model at the proximal tibia in rabbits (Chen et al., 2015). After anatomical reduction, the fracture was fixed with a compression screw. The authors reported intramembranous bone formation at the pre-existing bone trabeculae and already in the bone marrow $5 \mathrm{~d}$ after surgery. After 2 weeks, the fracture gap was filled with new bone trabeculae. The original bone structure was restored between week 4 and 8 after fracture. Also in this study, no signs of external callus formation and endochondral bone formation were

Table 3. Rabbit models for metaphyseal fracture healing.

\begin{tabular}{|c|c|c|c|c|c|c|c|}
\hline Strain & Age & Sex & $\begin{array}{c}\text { Fracture } \\
\text { site }\end{array}$ & Fracture model & $\begin{array}{c}\text { Osteoporosis } \\
\text { model }\end{array}$ & Healing outcome & Reference \\
\hline N/A & adult & N/A & $\begin{array}{l}\text { distal } \\
\text { femur }\end{array}$ & $\begin{array}{l}\text { incomplete } \\
\text { osteotomy, no } \\
\text { fixation }\end{array}$ & N/A & $\begin{array}{l}\text { appearance of } \\
\text { external callus } \\
\text { depends on } \\
\text { fracture stability }\end{array}$ & $\begin{array}{c}\text { Uhthoff et al., } \\
1981\end{array}$ \\
\hline $\begin{array}{l}\text { New } \\
\text { Zealand } \\
\text { white }\end{array}$ & $\begin{array}{l}16-48 \\
\text { weeks }\end{array}$ & either & $\begin{array}{l}\text { distal } \\
\text { femur }\end{array}$ & $\begin{array}{l}\text { open transverse } \\
\text { osteotomy } \\
\text { (0.15 mm gap), } \\
\text { fixation by } \\
\text { intramedullary } \\
\text { PLLA rod and } \\
\text { PGA membrane }\end{array}$ & N/A & $\begin{array}{l}\text { healing by } \\
\text { periosteal bone } \\
\text { formation }\end{array}$ & $\begin{array}{c}\text { Ashammakhi et } \\
\text { al., } 1994\end{array}$ \\
\hline $\begin{array}{l}\text { Japanese } \\
\text { white }\end{array}$ & $\begin{array}{c}6 \\
\text { months }\end{array}$ & N/A & $\begin{array}{c}\text { proximal } \\
\text { tibia }\end{array}$ & $\begin{array}{l}\text { bilateral, open } \\
\text { osteotomy, } \\
\text { fixation by a } \\
\text { K-wire staple }\end{array}$ & N/A & $\begin{array}{l}\text { healing by } \\
\text { intramembranous } \\
\text { bone formation, } \\
\text { small external } \\
\text { callus }\end{array}$ & Chen et al., 2004 \\
\hline $\begin{array}{l}\text { New } \\
\text { Zealand } \\
\text { white }\end{array}$ & $\begin{array}{l}1.5 \\
\text { months }\end{array}$ & male & $\begin{array}{l}\text { proximal } \\
\text { fibula }\end{array}$ & $\begin{array}{l}\text { open osteotomy } \\
(0.5 \mathrm{~mm} \text { gap })\end{array}$ & N/A & $\begin{array}{l}\text { healing by } \\
\text { intramembranous } \\
\text { bone formation }\end{array}$ & $\begin{array}{l}\text { Matos et al., } \\
\quad 2007\end{array}$ \\
\hline $\begin{array}{l}\text { New } \\
\text { Zealand } \\
\text { white }\end{array}$ & $\begin{array}{l}16-18 \\
\text { weeks }\end{array}$ & female & $\begin{array}{l}\text { distal } \\
\text { tibia }\end{array}$ & $\begin{array}{l}\text { open, unicortical, } \\
\text { wedge-shaped } \\
\text { osteotomy (lateral } \\
\text { gap size } 4 \mathrm{~mm} \text { ), } \\
\text { fixation by } \\
\text { external fixator }\end{array}$ & N/A & $\begin{array}{l}\text { healing by external } \\
\text { callus formation }\end{array}$ & $\begin{array}{l}\text { Tsiridis et al., } \\
2007 \\
\text { Morgan et al., } \\
2008\end{array}$ \\
\hline $\begin{array}{l}\text { New } \\
\text { Zealand } \\
\text { white }\end{array}$ & $\begin{array}{c}6 \\
\text { months }\end{array}$ & N/A & $\begin{array}{c}\text { proximal } \\
\text { tibia }\end{array}$ & $\begin{array}{l}\text { bilateral, oblique } \\
\text { fracture through } \\
\text { the tibial plateau, } \\
\text { fixation by } \\
\text { compression } \\
\text { screw }\end{array}$ & N/A & $\begin{array}{l}\text { healing by } \\
\text { intramembranous } \\
\text { bone formation }\end{array}$ & Han et al., 2012 \\
\hline $\begin{array}{l}\text { New } \\
\text { Zealand } \\
\text { white }\end{array}$ & $\begin{array}{c}8 \\
\text { months }\end{array}$ & female & $\begin{array}{l}\text { distal } \\
\text { femur }\end{array}$ & $\begin{array}{l}\text { open, half-round } \\
\text { fracture } 10 \mathrm{~mm} \\
\text { proximal to the } \\
\text { distal femur } \\
\text { end, fixation by } \\
\text { different tubes } \\
\text { and bone cements }\end{array}$ & $\begin{array}{l}\text { osteoporosis } \\
\text { by ovariectomy } \\
\text { and } \\
\text { glucocorticoid } \\
\text { treatment }\end{array}$ & $\begin{array}{l}\text { healing by } \\
\text { intramembranous } \\
\text { bone formation }\end{array}$ & $\begin{array}{l}\text { Nishizuka et al. } \\
2014\end{array}$ \\
\hline $\begin{array}{l}\text { New } \\
\text { Zealand } \\
\text { white }\end{array}$ & $\begin{array}{c}6 \\
\text { months }\end{array}$ & N/A & $\begin{array}{c}\text { proximal } \\
\text { tibia }\end{array}$ & $\begin{array}{c}\text { open vertical } \\
\text { osteotomy at } \\
\text { the medial tibial } \\
\text { plateau, fixation } \\
\text { by compression } \\
\text { screw }\end{array}$ & N/A & $\begin{array}{c}\text { healing by } \\
\text { intramembranous } \\
\text { bone formation }\end{array}$ & Chen et al., 2015 \\
\hline
\end{tabular}


found throughout the whole fracture healing process. Another study established a proximal tibia osteotomy model which was stabilised by K-wires (Chen et al., 2004). Also in this model, all fractures healed without pronounced external callus formation. Histological analysis revealed both intramembranous and endochondral bone formation in the fracture area, indicating higher interfragmentary tissue strains. In this study, fractures healed between 4 and 8 weeks after surgery. In summary, several models were described at the proximal tibia in rabbits using different fracture fixation devices, resulting in different degrees of mechanical stability and therefore different fracture healing modes.

Many studies using rabbit for metaphyseal fracture healing research are conducted on the distal femur. To investigate the influence of fracture geometry and gap size, Uhthoff et al. performed an incomplete osteotomy on the rabbit distal femur (Uhthoff and Rahn, 1981). No fracture fixation was used. Gap sizes varied greatly and, in some animals, spontaneous fractures occurred during the experimental period. Histological analysis revealed that the healing course was highly dependent on the stability of the fractures. Rabbits with spontaneous fractures displayed external callus formation and endochondral ossification at the fracture site. More stable fractures healed by intramembranous ossification, without external callus formation, within 3 weeks. This model lacks standardisation and cannot be recommended for ethical reasons. Ashammakhi et al. published a complete metaphyseal osteotomy model (Ashammakhi et al., 1994). They sawed a $0.15 \mathrm{~mm}$ osteotomy gap in the distal femur metaphysis and stabilised the fracture by using an intramedullary rod. However, the authors reported a high rate of adverse events, both during the surgery and the post-operative time course, due to implant failure - indicating an insufficient fracture stabilisation in this model. Nishizuka et al. tested a different fixation material for an incomplete, half-round fracture of the rabbit distal femur (Nishizuka et al., 2014). They used OVX rabbits treated with glucocorticoids to induce severe osteoporosis. Fractures were stabilised with different configurations. Dependent on the used fracture fixation device, there was a high rate of adverse events after surgery, indicating the need for a well-controlled model to study metaphyseal fracture healing at the rabbit distal femur.

In conclusion, several rabbit models were developed to study metaphyseal fracture healing at different fracture locations, with different fracture geometries and fixations. Healing occurred within 4 to 8 weeks. Both intramembranous ossification and endochondral ossification were found, dependent on the stability of the used fracture configuration. Some studies showed a high rate of adverse events due to implant failure, indicating the need to further improve metaphyseal fracture models in rabbits.

\section{Rats}

For metaphyseal fracture healing research, rats have been most commonly used. The models are located either at the proximal tibia or the distal femur, but vary widely regarding fracture geometry and stabilisation. Closed and open model were used with and without fixation devices (Table 4).

Aspenberg et al. proposed a very simple model to study metaphyseal bone healing (Meunier and Aspenberg, 2006). The group created a unicortical drill hole into the trabecular bone at the medial site of the proximal tibia and inserted a screw. Bone formation was analysed histologically, and the interfacial shear strength was measured using a pull-out test. The pull-out force increased during the experimental time period of $14 \mathrm{~d}$, indicating bony integration of the implant. This model may be very simple and highly standardised; however, it reflects osseointegration of an implant rather than metaphyseal healing normally occurring between two bony fragments. Nevertheless, the authors used this model in many further studies to investigate the effects of unloading and various treatments, such as cyclooxygenase 2 (Cox2) inhibition, PTH, bisphosphonates, Dickkopf-1 inhibition, tissue necrosis factor $\alpha$ inhibition, Sclerostin inhibition and receptor activator of the $\mathrm{NF}-\kappa \mathrm{B}$ ligand (RANKL) inhibition on trabecular bone healing (Agholme et al., 2011; Agholme et al., 2014; Aspenberg et al., 2008; Bernhardsson et al., 2015a; Bernhardsson et al., 2015b; Meunier and Aspenberg, 2006; Sandberg et al., 2012; Sandberg et al., 2014). The model was also used to compare metaphyseal and diaphyseal fracture healing in rats (Bernhardsson et al., 2015b; Sandberg and Aspenberg, 2015a). These latter studies demonstrated that the inflammatory response differs between the two type of fractures, whereas both follow similar biological principles regarding osteoanabolic pathways.

Jarry et al. (Jarry and Uhthoff, 1971) used different "real" fracture models at the distal femur and the proximal tibia. To study the influence of the fracture type and of biomechanical stimuli, the authors created closed fractures or performed osteotomies in an open approach. The fracture was left unfixed or stabilised by an intramedullary K-wire. Some experimental groups were immobilised. The main outcome of this study was that external callus formation and endochondral ossification was found under mechanically unstable conditions, whereas intramembranous bone healing without periosteal callus formation was observed in more stable situations. This indicates that, also in the rat, metaphyseal fracture healing is highly dependent on the mechanical environment. However, the mechanical conditions can hardly be controlled in the models used and may vary highly within the different groups, even after fixation of the fracture with an intramedullary pin - which does not provide rotational and shear stability (Histing et al., 2011). 
Table 4. Rat models for metaphyseal fracture healing.

\begin{tabular}{|c|c|c|c|c|c|c|c|}
\hline Strain & Age & Sex & $\begin{array}{c}\text { Fracture } \\
\text { site }\end{array}$ & Fracture model & $\begin{array}{c}\text { Osteoporosis } \\
\text { model }\end{array}$ & Healing outcome & References \\
\hline $\begin{array}{l}\text { Sprague- } \\
\text { Dawley }\end{array}$ & N/A & N/A & $\begin{array}{l}\text { distal } \\
\text { femur or } \\
\text { proximal } \\
\text { tibia }\end{array}$ & $\begin{array}{c}\text { open and closed } \\
\text { fractures/ } \\
\text { osteotomies, } \\
\text { fixation by K-wire } \\
\text { or no fixation }\end{array}$ & N/A & $\begin{array}{l}\text { appearance of } \\
\text { external callus } \\
\text { depends on } \\
\text { fracture stability }\end{array}$ & Jarry et al., 1971 \\
\hline N/A & adult & N/A & $\begin{array}{l}\text { distal } \\
\text { femur }\end{array}$ & $\begin{array}{l}\text { open, oblique } \\
\text { osteotomy, no } \\
\text { fixation }\end{array}$ & N/A & $\begin{array}{l}\text { appearance of } \\
\text { external callus } \\
\text { depends on } \\
\text { fracture stability }\end{array}$ & $\begin{array}{c}\text { Uhthoff et al., } \\
1981\end{array}$ \\
\hline $\begin{array}{l}\text { Sprague- } \\
\text { Dawley }\end{array}$ & $\begin{array}{c}\text { 8-20 } \\
\text { weeks }\end{array}$ & either & $\begin{array}{l}\text { proximal } \\
\text { tibia }\end{array}$ & $\begin{array}{c}\text { open, unicortical } \\
\text { drill hole defect, } \\
\text { inserted screw (1.7 } \\
\text { mm diameter, } 3 \\
\text { mm length) }\end{array}$ & $\begin{array}{l}\text { unloading } \\
\text { by muscle } \\
\text { paralysis }\end{array}$ & $\begin{array}{l}\text { healing by } \\
\text { intramembranous } \\
\text { bone formation }\end{array}$ & $\begin{array}{c}\text { Meunier et al., } \\
2006 \\
\text { Aspenberg et al., } \\
2008 \\
\text { Agholme et al., } \\
2011 \\
\text { Sandberg et al., } \\
2012 \\
\text { Agholme et al., } \\
2013 \\
\text { Sandberg et al., } \\
2014 \\
\text { Bernhardsson et } \\
\text { al., } 2015\end{array}$ \\
\hline Wistar & $\begin{array}{c}20 \\
\text { weeks }\end{array}$ & male & $\begin{array}{l}\text { distal } \\
\text { femur }\end{array}$ & $\begin{array}{l}\text { open, transverse } \\
\text { transcondylar } \\
\text { osteotomy, fixation } \\
\text { with PLLA-rod }\end{array}$ & N/A & $\begin{array}{l}\text { healing by } \\
\text { periosteal callus } \\
\text { formation }\end{array}$ & $\begin{array}{l}\text { Handolin et al., } \\
2007\end{array}$ \\
\hline Wistar & $\begin{array}{c}7-9 \\
\text { months }\end{array}$ & female & $\begin{array}{l}\text { proximal } \\
\text { tibia }\end{array}$ & $\begin{array}{l}\text { open, complete } \\
\text { mid-sagittal } \\
\text { osteotomy, fixation } \\
\text { with cerclage }\end{array}$ & $\begin{array}{l}\text { ovariectomy } \\
\text { (OVX), ageing, } \\
\text { glucocorticoid- } \\
\text { induced } \\
\text { osteoporosis }\end{array}$ & $\begin{array}{l}\text { healing by } \\
\text { intramembranous } \\
\text { bone formation }\end{array}$ & $\begin{array}{c}\text { Nozaka et al., } 2008 \\
\text { Aonuma et al., } \\
2011 \\
\text { Aonuma et al., } \\
2014 \\
\text { Kawano et al., } \\
2017 \\
\end{array}$ \\
\hline $\begin{array}{l}\text { Sprague- } \\
\text { Dawley }\end{array}$ & \begin{tabular}{|c|}
12 \\
weeks \\
-11 \\
months
\end{tabular} & either & $\begin{array}{l}\text { proximal } \\
\text { tibia }\end{array}$ & $\begin{array}{l}\text { open osteotomy } \\
\text { (gap } 0.5 \mathrm{~mm} \text { ), } \\
\text { fixation by five-hole } \\
\text { T-shaped miniplate }\end{array}$ & $\begin{array}{l}\text { OVX, } \\
\text { orchidectomy } \\
\text { (ORX) }\end{array}$ & $\begin{array}{l}\text { healing by } \\
\text { intramembranous } \\
\text { bone formation } \\
\text { with small } \\
\text { periosteal callus } \\
\text { in the absence of } \\
\text { fibula fractures; } \\
\text { large periosteal } \\
\text { callus formation } \\
\text { in the presence of } \\
\text { fibula fractures }\end{array}$ & $\begin{array}{c}\text { Stuermer et al., } \\
2010 \\
\text { Kolios et al., } 2010 \\
\text { Komrakova et al., } \\
2010 \\
\text { Komrakova et al., } \\
2011 \\
\text { Komrakova et al., } \\
2013 \\
\text { Stuermer } \text { et al., } \\
2014 \\
\text { Komrakova et al., } \\
2015 \\
\text { Saul et al., } 2018 \\
\end{array}$ \\
\hline $\begin{array}{l}\text { Sprague- } \\
\text { Dawley }\end{array}$ & $\begin{array}{c}26 \\
\text { weeks, } \\
7-8 \\
\text { months }\end{array}$ & female & $\begin{array}{l}\text { distal } \\
\text { femur }\end{array}$ & $\begin{array}{l}\text { open wedge-shaped } \\
\text { osteotomy (lateral } \\
\text { height of } 3,4 \text { or } 5 \\
\text { mm, medial height } \\
1 \mathrm{~mm} \text { ), fixation by } \\
\text { 7-hole T-shaped } \\
\text { miniplate }\end{array}$ & $\begin{array}{c}\text { OVX and } \\
\text { multi-deficient } \\
\text { diet }\end{array}$ & $\begin{array}{l}\text { osteotomy with } 5 \\
\text { mm lateral height } \\
\text { displayed non- } \\
\text { union formation, } \\
\text { healing of smaller } \\
\text { defects by } \\
\text { intramembranous } \\
\text { bone formation } \\
\text { with small } \\
\text { periosteal callus } \\
\text { formation }\end{array}$ & $\begin{array}{c}\text { Alt et al., } 2013 \\
\text { Thormann et al., } \\
2013 \\
\text { Lips et al., } 2013 \\
\text { Lips et al., } 2014 \\
\text { Thormann et al., } \\
2014 \\
\text { Kampschulte et } \\
\text { al., } 2016 \\
\text { Ray et al., } 2016 \\
\text { Ray et al., } 2018 \\
\end{array}$ \\
\hline $\begin{array}{l}\text { Sprague- } \\
\text { Dawley }\end{array}$ & $\begin{array}{c}12 \\
\text { weeks }\end{array}$ & female & $\underset{\text { tibia }}{\text { proximal }}$ & $\begin{array}{c}\text { open osteotomy } \\
\text { (gap } 1 \mathrm{~mm}), \\
\text { fixation by five-hole } \\
\text { Y-shaped miniplate }\end{array}$ & OVX & $\begin{array}{l}\text { healing by } \\
\text { periosteal callus } \\
\text { formation }\end{array}$ & $\begin{array}{c}\text { Mackert et al., } \\
2017\end{array}$ \\
\hline
\end{tabular}


Further metaphyseal fracture models were established in the distal rat femur. After creating an oblique osteotomy, Uhthoff et al. observed exclusively intramembranous bone formation, indicating a high degree of mechanical stability, although no fracture fixation device was used (Uhthoff and Rahn, 1981). In this model, the bones were completely healed within a period of $42 \mathrm{~d}$. Despite being rather simple from a technical aspect, this model may provide a relatively high degree of standardisation. Handolin et al. performed a transverse transcondylar osteotomy, which was stabilised with an intramedullary rod (Handolin et al., 2007). The authors described periosteal callus formation and intramembranous ossification at the fracture site. All osteotomies were bridged with newly formed bone after 12 weeks. However, $10 \%$ of the animals displayed implant failure and were excluded from the study. This model was further used to analyse the influence of low-intensity pulsed ultrasound on metaphyseal fracture healing. Its disadvantages are the high rate of adverse events and the relatively long healing time. Alt et al. established a critical size osteotomy model with the primary aim of testing bone grafts to support metaphyseal bone healing (Alt et al., 2013). They created a complete wedge-shaped osteotomy at the distal femur with a lateral gap size of 3 to $5 \mathrm{~mm}$ and stabilised the fragments with a commercially available T-shaped miniplate. Whereas the rats with the $3 \mathrm{~mm}$ gap healed completely, the $5 \mathrm{~mm}$ osteotomies did not heal after 6 weeks, indicating a critical size for this defect. The authors also used this model to demonstrate that metaphyseal bone healing is impaired in ovariectomised, osteoporotic rats (Thormann et al., 2014). This model is well standardised, allows variation of the gap size and can further be used to study the effect of inserting biomaterials into the gap (Kampschulte et al., 2016; Lips et al., 2013; Lips et al., 2014; Ray et al., 2018; Ray et al., 2016; Thormann et al., 2013).

Besides the distal femur, many studies selected the proximal tibia as a fracture location. Nozaka et al. created a vertical osteotomy model at the proximal tibia in ovariectomised, osteoporotic rats (Nozaka et al., 2008). A mid-sagittal osteotomy was sawed from the joint surface to the diaphysis of the tibia. The osteotomy was fixed with a circumferential cerclage. The osteoporotic rats displayed reduced bone formation compared to non-OVX animals. $40 \%$ and $27 \%$ of bony union was observed in the control and osteoporotic rats, respectively, after 5 weeks. This model was further used to investigate the effects of low intensity pulsed ultrasound (LIPUS), alendronate and PTH on metaphyseal fracture healing in OVX or aged rats or in rats with glucocorticoid-induced osteoporosis (Aonuma et al., 2011; Aonuma et al., 2014; Kawano et al., 2017). A disadvantage of this model is the relatively long healing period, as most of the fractures were not healed after 5 weeks.

Stuermer et al. introduced a transverse osteotomy model at the proximal tibia (Stuermer et al., 2010b).
An osteotomy with a gap size of $0.5 \mathrm{~mm}$ was created $7 \mathrm{~mm}$ distal to the tibial plateau. The fracture was stabilised by a commercially available T-shaped miniplate. Some animals, which accidentally suffered from additional fibula fractures, developed a large external fracture callus at the tibia due to the unstable mechanical situation and had to be excluded from the study. The rats with isolated tibial fractures displayed mainly endosteal healing with moderate periosteal callus formation. The fractures were bridged with bone after $35 \mathrm{~d}$. This model is well standardised, but the drop-out rate is relatively high due to additional fibula fractures, indicating that the operating procedure is probably challenging. The model was used in further studies to investigate the effects of oestrogen, oestrogen-receptor modulators, $\mathrm{PTH}$, alendronate, strontium ranelate, zileuton and whole-body vibration on osteoporotic metaphyseal fracture healing (Kolios et al., 2010a; Kolios et al., 2010b; Kolios et al., 2009; Komrakova et al., 2011a; Komrakova et al., 2013; Komrakova et al., 2011b; Komrakova et al., 2010; Komrakova et al., 2015; Saul et al., 2018; Stuermer et al., 2014; Stuermer et al., 2010a; Stuermer et al., 2010b). In later studies, the authors even used bilateral tibial osteotomies. A very similar fracture model was recently used to evaluate the effects of an extracorporeal shockwave therapy on metaphyseal fracture healing in ovariectomised rats. These authors created a $1 \mathrm{~mm}$ osteotomy gap and used a Y-shaped miniplate for fracture fixation (Mackert et al., 2017). Others used a similar model, but with a smaller gap size $(0.35 \mathrm{~mm})$ to investigate the effects of a vibration treatment on metaphyseal fracture healing in osteoporotic rats (Choy et al., 2020; Wong et al., 2019).

Concluding, various rat models with different fracture geometries and fixation techniques were established for use at the distal femur or the proximal tibia. The fracture healing time varied between $35 \mathrm{~d}$ and 12 weeks, dependent on the model. Many rat studies used osteoporosis models to study osteoporotic metaphyseal fracture healing; however, variability was rather high especially in osteoporotic animals.

\section{Mice}

Despite their small skeleton, which makes operative procedures challenging, mice are increasingly used for metaphyseal fracture healing research (Fig. 1). 11 studies were found in PMC, all published within the last 8 years (Table 5). The fracture models vary widely, but similar to the rat studies, the most common fracture locations are the distal femur and the proximal tibia. Only one study used the proximal femur as a more clinically relevant fracture location.

The first study to use mice for metaphyseal fracture healing research was published by Histing et al. (2012). The authors conducted an open transverse osteotomy with a $0.22 \mathrm{~mm}$ gap size at the distal femur, stabilised by a commercially available 3-hole locking plate designed specifically for mice. The authors 
observed no adverse events during the experimental time period. In all animals, the plates and osteotomies were located correctly, indicating technical feasibility. After 5 weeks, all fractures were almost completely healed. Biomechanical testing revealed a significantly higher rotational stiffness 5 weeks after fracture $(90 \%$ compared to unfractured bone) compared to 2 weeks (30\%). Histological analysis demonstrated that the fractures healed without external callus formation by intramembranous ossification, indicating high mechanical stability. This model seems to provide a high degree of standardisation. A disadvantage might be that it is technically difficult to fix a plate on the small mouse bone. This requires experienced surgeons. A very similar fracture model but with a large gap size of $1.2 \mathrm{~mm}$ was used for a further study, in which a bone graft was tested in osteoporotic mice (Kauschke et al., 2018).

Another fracture model at the distal femur was published by Han et al. (Han et al., 2015). The authors created an intercondylar fracture by splitting the bone with a scalpel. The fractures were stable without any fixation, but the authors reported that the fracture geometry varied between the animals. Most fractures already displayed bony bridging after $7 \mathrm{~d}$. The authors reported that fractures healed by intramembranous bone formation. Existing bone trabeculae were activated, and then redundant bone was resorbed by osteoclasts. Disadvantages of this model are the lack of standardisation and the clinically rather rare fracture type.

The simplest model for studying metaphyseal bone healing at the proximal tibia of mice was proposed by the Aspenberg et al. group, according to their abovementioned rat studies (Meunier and Aspenberg, 2006). A unicortical drill hole of $0.6 \mathrm{~mm}$ diameter was created in the metaphyseal bone of the proximal tibia and a $0.7 \mathrm{~mm}$-diameter and $1 \mathrm{~mm}$-length screw was inserted. The screws were firmly integrated into the bone within 2 weeks. The new bone in the interface was formed by intramembranous ossification. The disadvantage of this model was already mentioned above. It does not really reflect metaphyseal fracture healing but rather osseointegration of an artificial implant surface. The group used this mouse model to recapitulate some of their rat studies and investigated the effects of glucocorticoids, alendronate and PTH on trabecular bone healing (Bernhardsson and Aspenberg, 2018; Sandberg and Aspenberg, 2015a; Sandberg et al., 2017). Furthermore, the immune reaction towards the bone injury was analysed in this model as well as the effects of this fracture type on the

Table 5. Mouse models for metaphyseal fracture healing.

\begin{tabular}{|c|c|c|c|c|c|c|c|}
\hline Strain & Age & Sex & $\begin{array}{c}\text { Fracture } \\
\text { site }\end{array}$ & Fracture model & $\begin{array}{c}\text { Osteoporosis } \\
\text { model }\end{array}$ & Healing outcome & References \\
\hline $\begin{array}{c}\text { CD-1, } \\
\text { B6.129S6- } \\
\text { CF1-Chrm- } \\
3^{\text {tm1Jwe }}\end{array}$ & $\begin{array}{c}12-16 \\
\text { weeks }\end{array}$ & female & $\begin{array}{l}\text { distal } \\
\text { femur }\end{array}$ & $\begin{array}{c}\text { open transverse } \\
\text { osteotomy } \\
(0.22 \mathrm{~mm} \text { or } \\
1.2 \mathrm{~mm} \text { gap), } \\
\text { fixation by 3-hole } \\
\text { locking plate }\end{array}$ & $\begin{array}{c}\text { osteoporosis } \\
\text { induced by } \\
\text { M3 mAChR } \\
\text { KO }\end{array}$ & $\begin{array}{l}\text { healing by } \\
\text { intramembranous } \\
\text { bone formation }\end{array}$ & $\begin{array}{l}\text { Histing et al., } \\
2012 \\
\text { Kauschke et } \\
\text { al., } 2018\end{array}$ \\
\hline C57BL/6 & $\begin{array}{c}24 \\
\text { weeks }\end{array}$ & N/A & $\begin{array}{c}\text { proximal } \\
\text { tibia }\end{array}$ & $\begin{array}{l}\text { open transverse } \\
\text { osteotomy, fixation } \\
\text { by } 27 \mathrm{G} \text { needle }\end{array}$ & N/A & $\begin{array}{l}\text { healing by } \\
\text { external callus and } \\
\text { endochondral bone } \\
\text { formation }\end{array}$ & Tu et al., 2014 \\
\hline Balb/c & $\begin{array}{c}8-10 \\
\text { weeks }\end{array}$ & male & $\begin{array}{l}\text { distal } \\
\text { femur }\end{array}$ & $\begin{array}{l}\text { open, intercondylar } \\
\text { fracture, no } \\
\text { fixation }\end{array}$ & N/A & $\begin{array}{c}\text { healing by } \\
\text { intramembranous } \\
\text { bone formation }\end{array}$ & $\begin{array}{l}\text { Han et al., } \\
\quad 2015\end{array}$ \\
\hline C57BL/6 & $\begin{array}{c}10 \\
\text { weeks }\end{array}$ & either & $\begin{array}{c}\text { proximal } \\
\text { tibia }\end{array}$ & $\begin{array}{l}\text { open, unicortical } \\
\text { drill hole defect, } \\
\text { inserted screw } \\
(1 \mathrm{~mm} \text { length, } \\
0.7 \mathrm{~mm} \text { diameter })\end{array}$ & N/A & $\begin{array}{l}\text { healing by } \\
\text { intramembranous } \\
\text { bone formation }\end{array}$ & $\begin{array}{c}\text { Sandberg et } \\
\text { al., } 2015 \\
\text { Sandberg et } \\
\text { al., 2016 } \\
\text { Tätting et al., } \\
2017 \\
\text { Bernhardsson } \\
\text { et al., } 2018 \\
\text { Tätting et al., } \\
2018\end{array}$ \\
\hline C57BL/6J & $\begin{array}{c}12 \\
\text { weeks }\end{array}$ & female & $\begin{array}{l}\text { proximal } \\
\text { femur }\end{array}$ & $\begin{array}{l}\text { open, transverse } \\
\text { osteotomy between } \\
\text { the third and lesser } \\
\text { trochanter ( } 0.4 \mathrm{~mm} \\
\text { gap size), fixation } \\
\text { by } 24 \mathrm{G} \text { needle }\end{array}$ & N/A & $\begin{array}{l}\text { healing by } \\
\text { external callus and } \\
\text { endochondral bone } \\
\text { formation }\end{array}$ & $\begin{array}{c}\text { Haffner- } \\
\text { Luntzer et al., } \\
2020\end{array}$ \\
\hline
\end{tabular}


intact skeleton (Tatting et al., 2017; Tatting et al., 2018). This model was also used to compare metaphyseal and diaphyseal fracture healing (Tatting et al., 2018).

Tu et al. (Tu et al., 2014) introduced a transverse osteotomy model at the proximal mouse tibia. Tibiae were stabilised by inserting a $27 \mathrm{G}$ needle into the intramedullary cavity. Displacement of the tibial fractures and additional fibula fractures were frequently observed. 4 weeks after surgery, most fractures displayed bony union. Fractures healed by external callus formation and endochondral ossification, indicating relatively high interfragmentary tissue strains. The disadvantages of this model are the low degree of standardisation and uncontrolled mechanical conditions due to the poor facture fixation.

The Institute of Orthopaedic Research and Biomechanics group at Ulm established a novel fracture model at the mouse proximal femur, because many osteoporotic fractures occur in this area in humans (Haffner-Luntzer et al., 2020). A transverse osteotomy between the third and the lesser trochanter was created using a $0.44 \mathrm{~mm}$ Gigli wire saw and fixed with an intramedullary a $24 \mathrm{G}$ needle. The mice tolerated the operation well and loaded the limb normally immediately after the operation. No adverse events were observed during the experimental time period. All animals displayed successful bony union at day 21 after surgery. Histological analysis revealed periosteal callus formation and endochondral ossification. Advantages of this model are the clinically relevant fracture location and the easy surgical protocol. Disadvantages are that the intramedullary needle does not provide rotational stability and that only few bone trabeculae are found in the metaphyseal region of the proximal mouse femur.

In summary, several metaphyseal fracture healing models have been described in mice. Most studies were conducted on the distal femur and the proximal tibia, one study investigated healing at the proximal femur. Fracture healing occurred between 1 and 5 weeks after surgery, dependent on the model. So far, there are no studies available comparing metaphyseal fracture healing in osteoporotic and non-osteoporotic mice in a translationally relevant model. Because mice are the best model to study molecular mechanisms, metaphyseal fracture healing models should be further refined.

\section{Differences between metaphyseal and diaphyseal fracture healing}

It is widely believed that metaphyseal bone heals without external callus formation by direct bone formation. In contrast, diaphyseal fracture healing is more likely to show periosteal callus formation and endochondral ossification. However, concluding from the different models reviewed here, preclinical data suggest that both intramembranous and endochondral bone formation can also occur in metaphyseal bone healing, dependent on interfragmentary strains acting at the fracture site, as it was shown for diaphyseal healing. Less stable fracture fixations (intramedullary needles/wires, cerclages) were more likely to heal by periosteal callus formation and endochondral ossification, whereas stable fracture fixations (plates, compression screws) or anatomically reduced fracture gaps mostly heal by direct bone formation without external callus formation. Indeed, Claes et al. demonstrated that metaphyseal fracture healing follows similar biomechanical rules to diaphyseal healing in sheep (Claes et al., 2011a). Also clinically, both types of bone formation can be found in metaphyseal bone depending on the fracture location, the fracture geometry and the fixation technique (Hinsenkamp et al., 1984). However, healing without pronounced external callus is more likely in metaphyseal than in diaphyseal fractures and metaphyseal fractures are considered to heal faster (McKibbin, 1978).

Several studies were conducted to compare metaphyseal and diaphyseal fracture healing with respect to biological factors. Significant differences were found between the two types of fractures. Diaphyseal fracture healing seems to be highly dependent on the activation of periosteal progenitor cells, even if other stem cell sources also contribute to bone formation (Blokhuis, 2017). In contrast, metaphyseal healing is less dependent on periosteal cells, since a higher number of active osteogenic cells are available at the existing trabeculae and in the bone marrow in between (Sandberg and Aspenberg, 2016). Furthermore, osteoblasts isolated from different locations display a different proliferation and differentiation capacity, which could contribute to the different healing capacities of different bone locations (Martinez et al., 1999). This was not only true for metaphyseal vs. diaphyseal bone, but also for different bones. Furthermore, blood flow and vascularisation were shown to be different in the diaphyseal and metaphyseal regions of the bone, with higher vascularisation in the metaphyseal bone (Blokhuis, 2017; Sandberg and Aspenberg, 2016). This indicates the fracture location might significantly impact the healing capacity and might account for the faster healing of metaphyseal fractures (McKibbin, 1978).

It was also suggested that the inflammatory response plays a crucial role in diaphyseal healing, but may be less important in metaphyseal bone healing (Tatting et al., 2018). Furthermore, metaphyseal fractures led to a rather acute inflammatory response, whereas diaphyseal fractures led to a more chronic inflammation. In line with that, anti-inflammatory drugs such as NSAIDs or glucocorticoids exert greater effects on diaphyseal than metaphyseal healing (Sandberg and Aspenberg, 2015a; Sandberg and Aspenberg, 2015b). However, attention has to be paid regarding these studies since a diaphyseal complete fracture model with higher interfragmentary 
movements and a higher degree of trauma was compared to a metaphyseal drill-hole model with almost no biomechanical stimulation and less tissue trauma. Further studies are necessary to compare similar boundary conditions in metaphyseal and diaphyseal bone to investigate potential differences in the inflammatory response.

Some studies stated that an antiresorptive treatment with bisphosphonates supports fracture healing, both in metaphyseal and diaphyseal bone (Kolios et al., 2010b; Morse et al., 2020; Sandberg et al., 2017). Several studies demonstrated that treatment with various anti-resorptive drugs lead to a larger callus with increased mechanical stiffness and strength, because of delayed external callus remodelling. Thus, it could be speculated that diaphyseal fractures, which mostly heal by secondary healing by means of external callus formation, might benefit to a greater extend from antiresorptive treatment that metaphyseal fractures.

Not surprisingly, osteoanabolic treatments support healing of both metaphyseal and diaphyseal fractures. This was shown for PTH, which increases osteoblast differentiation and bone formation independent of the fracture location (Bernhardsson and Aspenberg, 2018; Li et al., 2017; Nozaka et al., 2008). Also, the activation of the crucial osteoanabolic Wnt/ $\beta$-catenin signalling pathway, by antagonising the Wnt inhibitor Dkk1, accelerates both metaphyseal and diaphyseal fracture healing (Agholme et al., 2011; Jin et al., 2015).

To conclude, antiresorptive and osteoanabolic treatment strategies support both diaphyseal and metaphyseal healing. However, because direct and indirect bone formation are differentially regulated and contribute in different proportions to metaphyseal or diaphyseal healing, the effect size of the different interventions might be different in both conditions. However, comparative studies are widely missing.

\section{Summary}

Both large (sheep and dogs) and small (rabbits, rats and mice) animal models are available to study metaphyseal fracture healing. Mainly used fracture locations are the proximal tibia and distal femur; however, some studies also used proximal ulna (sheep), proximal fibula (rabbit), distal tibia (rabbit) and proximal femur (mouse). Various fracture fixation techniques have been developed for the different animal species and fracture locations. Healing times varies greatly between the species and the fracture models. Mice display the fastest metaphyseal fracture healing with bony union between 1 and 5 weeks, depending on fracture localisation and type, whereas dog and sheep fracture healing take the longest with 2 to 3 months healing time. Each model found in the literature has certain advantages and disadvantages; however, many lack standardisation resulting in a high variability of the healing outcome or poorly mimic the clinical situation. Furthermore, in some manuscripts, description of the surgical technique is not detailed enough to allow repetition of the experiments.

The animal studies reviewed here indicate that metaphyseal and diaphyseal fracture healing follow similar basic biological and biomechanical principles, even if they may display specific differences regarding the progenitor cell source, the involvement of inflammatory processes, or the contribution of different biological pathways. However, so far, comparative studies are rare. Most studies directly comparing diaphyseal to metaphyseal fracture healing used animal models with different trauma severities for both type of injuries. This might influence the type and course of fracture healing even more than the fracture location. Therefore, further investigations are needed to unravel mechanistic molecular and cellular differences.

The multiple preclinical studies reviewed here showed various healing patterns depending on the biomechanical conditions acting at the fracture site. Stable fracture situations induced intramembranous ossification, whereas endochondral bone formation and external callus formation was observed under unstable conditions. A standardised fracture fixation is important to avoid high intra-experimental variability in metaphyseal fracture healing studies.

Since metaphyseal fractures are very common in osteoporotic individuals, specific animal models to mimic osteoporotic metaphyseal fracture healing are desirable (Marmor et al., 2015). Several large and small animal models have been developed to compare non-osteoporotic and osteoporotic metaphyseal fracture healing. However, many studies demonstrated high variability, especially in osteoporotic animals, and lack detection of significant differences between non-osteoporotic and osteoporotic fracture healing. Therefore, there is the great need for further refinement of animal models for osteoporotic metaphyseal fracture healing because suitable preclinical models are essential for the development of new treatment strategies.

\section{Acknowledgements}

This work was conducted in the context of the Collaborative Research Centre CRC1149 supported from the German Research Foundation (project number 251293561: INST 40/599-1 and INST 40/4912).

\section{References}

Agholme F, Isaksson H, Kuhstoss S, Aspenberg P (2011) The effects of Dickkopf-1 antibody on metaphyseal bone and implant fixation under different loading conditions. Bone 48: 988-996. 
Agholme F, Macias B, Hamang M, Lucchesi J, Adrian MD, Kuhstoss S, Harvey A, Sato M, Aspenberg P (2014) Efficacy of a sclerostin antibody compared to a low dose of PTH on metaphyseal bone healing. J Orthop Res 32: 471-476.

Alagboso FI, Budak M, Sommer U, Ray S, Kaiser A, Kampschulte M, Henss A, Durselen L, Biehl C, Lips KS, Heiss C, Thormann U, Alt V (2019) Establishment of a clinically relevant large animal model to assess the healing of metaphyseal bone. Eur Cell Mater 37: 444-466.

Alt V, Miclau T (2016) Osteoporotic fractures - the biological perspective. Injury 47 Suppl 1: S1-2.

Alt V, Thormann U, Ray S, Zahner D, Durselen L, Lips K, El Khassawna T, Heiss C, Riedrich A, Schlewitz G, Ignatius A, Kampschulte M, von Dewitz H, Heinemann S, Schnettler R, Langheinrich A (2013) A new metaphyseal bone defect model in osteoporotic rats to study biomaterials for the enhancement of bone healing in osteoporotic fractures. Acta Biomater 9: 7035-7042.

An YH, Friedman RJ, Powers DL, Draughn RA, Latour RA, Jr. (1998) Fixation of osteotomies using bioabsorbable screws in the canine femur. Clin Orthop Relat Res 355: 300-311.

Aonuma H, Miyakoshi N, Kasukawa Y, Kamo K, Sasaki H, Tsuchie H, Segawa T, Shimada Y (2011) Combined treatment of alendronate and lowintensity pulsed ultrasound (LIPUS) increases bone mineral density at the cancellous bone osteotomy site in aged rats: a preliminary study. JNMA J Nepal Med Assoc 51: 171-175.

Aonuma H, Miyakoshi N, Kasukawa Y, Kamo K, Sasaki H, Tsuchie H, Segawa T, Shimada Y (2014) Effects of combined therapy of alendronate and lowintensity pulsed ultrasound on metaphyseal bone repair after osteotomy in the proximal tibia of aged rats. J Bone Miner Metab 32: 232-239.

Ashammakhi N, Makela A, Vihtonen K, Rokkanen P, Tormala P (1994) Absorbable membranes for bone repair: an experimental study on rabbits. Clin Mater 17: 113-118.

Aspenberg P, Wermelin K, Tengwall P, Fahlgren A (2008) Additive effects of PTH and bisphosphonates on the bone healing response to metaphyseal implants in rats. Acta Orthop 79: 111-115.

Beil FT, Barvencik F, Gebauer M, Seitz S, Rueger JM, Ignatius A, Pogoda P, Schinke T, Amling M (2010) Effects of estrogen on fracture healing in mice. J Trauma 69: 1259-1265.

Bernhardsson M, Aspenberg P (2018) Abaloparatide versus teriparatide: a head to head comparison of effects on fracture healing in mouse models. Acta Orthop 89: 674-677.

Bernhardsson M, Sandberg O, Aspenberg P (2015a) Anti-RANKL treatment improves screw fixation in cancellous bone in rats. Injury 46: 990-995.

Bernhardsson M, Sandberg O, Aspenberg P (2015b) Experimental models for cancellous bone healing in the rat. Acta Orthop 86: 745-750.
Bindl R, Oheim R, Pogoda P, Beil FT, Gruchenberg K, Reitmaier S, Wehner T, Calcia E, Radermacher P, Claes L, Amling M, Ignatius A (2013) Metaphyseal fracture healing in a sheep model of low turnover osteoporosis induced by hypothalamic-pituitary disconnection (HPD). J Orthop Res 31: 1851-1857.

Blokhuis TJ (2017) Management of traumatic bone defects: Metaphyseal versus diaphyseal defects. Injury 48 Suppl 1: S91-S93.

Chen WJ, Jingushi S, Aoyama I, Anzai J, Hirata G, Tamura M, Iwamoto Y (2004) Effects of FGF-2 on metaphyseal fracture repair in rabbit tibiae. J Bone Miner Metab 22: 303-309.

Chen WT, Han da C, Zhang PX, Han N, Kou YH, Yin XF, Jiang BG (2015) A special healing pattern in stable metaphyseal fractures. Acta Orthop 86: 238242.

Choy MV, Wong RM, Li MC, Wang BY, Liu XD, Lee W, Cheng JC, Chow SK, Cheung WH (2020) Can we enhance osteoporotic metaphyseal fracture healing through enhancing ultrastructural and functional changes of osteocytes in cortical bone with low-magnitude high-frequency vibration? FASEB J 34: 4234-4252.

Claes L (2017) [Mechanobiology of fracture healing part 1 : Principles]. Unfallchirurg 120: 14-22.

Claes L, Reusch M, Gockelmann M, Ohnmacht M, Wehner T, Amling M, Beil FT, Ignatius A (2011a) Metaphyseal fracture healing follows similar biomechanical rules as diaphyseal healing. J Orthop Res 29: 425-432.

Claes L, Reusch M, Wolfram U, Göckelmann M, Ignatius A (2011b) Modelle der metaphysären Frakturheilung. Osteologie 20: 29-33.

Claes L, Veeser A, Gockelmann M, Simon U, Ignatius A (2009) A novel model to study metaphyseal bone healing under defined biomechanical conditions. Arch Orthop Trauma Surg 129: 923-928.

Fan W, Crawford R, Xiao Y (2008) Structural and cellular differences between metaphyseal and diaphyseal periosteum in different aged rats. Bone 42: 81-89.

Haffner-Luntzer M, Fischer V, Prystaz K, Liedert A, Ignatius A (2017) The inflammatory phase of fracture healing is influenced by oestrogen status in mice. Eur J Med Res 22: 23.

Haffner-Luntzer M, Weber B, Lam C, Fischer V, Lackner I, Ignatius A, Kalbitz M, Marcucio RS, Miclau $\mathrm{T}$ (2020) A novel mouse model to study fracture healing of the proximal femur. J Orthop Res.

Han D, Han N, Chen Y, Zhang P, Jiang B (2015) Healing of cancellous fracture in a novel mouse model. Am J Transl Res 7: 2279-2290.

Han N, Zhang PX, Wang WB, Han DC, Chen JH, Zhan HB, Jiang BG (2012) A new experimental model to study healing process of metaphyseal fracture. Chin Med J (Engl) 125: 676-679.

Handolin L, Partio EK, Arnala I, Pajarinen J, Patiala H, Rokkanen P (2007) The effect of lowintensity pulsed ultrasound on bone healing in SR- 
PLLA rod fixed experimental distal femur osteotomy in rat. J Mater Sci Mater Med 18: 1239-1245.

Heiss C, Schettler N, Wenisch S, Cords S, Schilke F, Lips KS, Alt V, Schnettler R (2010) Bond strength of an alkylene bis(dilactoyl)-methacrylate bone adhesive: a biomechanical evaluation in sheep. J Biomater Sci Polym Ed 21: 1345-1358.

Hinsenkamp M, Burny F, Andrianne Y, Quintin J, Rasquin C, Donkerwolcke M, Picchio AA, Asche G (1984) External fixation of the fracture of the humerus: a review of 164 cases. Orthopedics 7: 1309-1314.

Histing T, Garcia P, Holstein JH, Klein M, Matthys R, Nuetzi R, Steck R, Laschke MW, Wehner T, Bindl R, Recknagel S, Stuermer EK, Vollmar B, Wildemann B, Lienau J, Willie B, Peters A, Ignatius A, Pohlemann T, Claes L, Menger MD (2011) Small animal bone healing models: standards, tips, and pitfalls results of a consensus meeting. Bone 49: 591-599.

Histing T, Klein M, Stieger A, Stenger D, Steck R, Matthys R, Holstein JH, Garcia P, Pohlemann T, Menger MD (2012) A new model to analyze metaphyseal bone healing in mice. J Surg Res 178: 715-721.

Jarry L, Uhthoff HK (1971) Differences in healing of metaphyseal and diaphyseal fractures. Can J Surg 14: 127-135.

Jin H, Wang B, Li J, Xie W, Mao Q, Li S, Dong F, Sun Y, Ke HZ, Babij P, Tong P, Chen D (2015) AntiDKK1 antibody promotes bone fracture healing through activation of beta-catenin signaling. Bone 71: 63-75.

Kampschulte M, Krombach GA, Richards DC, Sender J, Lips KS, Thormann U, El Khassawna T, Ray S, Alt V, Langheinrich AC (2016) Neovascularization of osteoporotic metaphyseal bone defects: A morphometric micro-CT study. Microvasc Res 105: 7-14.

Kauschke V, Schneider M, Jauch A, Schumacher M, Kampschulte M, Rohnke M, Henss A, Bamberg C, Trinkaus K, Gelinsky M, Heiss C, Lips KS (2018) Effects of a pasty bone cement containing brain-derived neurotrophic factor-functionalized mesoporous bioactive glass pparticles on metaphyseal healing in a new murine osteoporotic fracture model. Int J Mol Sci 19: 3531. doi: 10.3390/ijms19113531

Kawano T, Miyakoshi N, Kasukawa Y, Hongo M, Tsuchie H, Sato C, Fujii M, Suzuki M, Akagawa M, Ono Y, Yuasa Y, Nagahata I, Shimada Y (2017) Effects of combined therapy of alendronate and low-intensity pulsed ultrasound on metaphyseal bone repair after osteotomy in the proximal tibia of glucocorticoid-induced osteopenia rats. Osteoporos Sarcopenia 3: 185-191.

Kolios L, Daub F, Sehmisch S, Frosch KH, Tezval M, Stuermer KM, Wuttke W, Stuermer EK (2010a) Absence of positive effect of black cohosh (Cimicifuga racemosa) on fracture healing in osteopenic rodent model. Phytother Res 24: 1796-1806.

Kolios L, Hoerster AK, Sehmisch S, Malcherek MC, Rack T, Tezval M, Seidlova-Wuttke D, Wuttke W, Stuermer KM, Stuermer EK (2010b) Do estrogen and alendronate improve metaphyseal fracture healing when applied as osteoporosis prophylaxis? Calcif Tissue Int 86: 23-32.

Kolios L, Sehmisch S, Daub F, Rack T, Tezval M, Stuermer KM, Stuermer EK (2009) Equol but not genistein improves early metaphyseal fracture healing in osteoporotic rats. Planta Med 75: 459-465.

Komrakova M, Krischek C, Wicke M, Sehmisch S, Tezval M, Rohrberg M, Brandsch T, Stuermer KM, Stuermer EK (2011a) Influence of intermittent administration of parathyroid hormone on muscle tissue and bone healing in orchiectomized rats or controls. J Endocrinol 209: 9-19.

Komrakova M, Sehmisch S, Tezval M, Ammon J, Lieberwirth P, Sauerhoff C, Trautmann L, Wicke M, Dullin C, Stuermer KM, Stuermer EK (2013) Identification of a vibration regime favorable for bone healing and muscle in estrogen-deficient rats. Calcif Tissue Int 92: 509-520.

Komrakova M, Sehmisch S, Tezval M, Schmelz U, Frauendorf H, Grueger T, Wessling T, Klein C, Birth M, Stuermer KM, Stuermer EK (2011b) Impact of 4-methylbenzylidene camphor, daidzein, and estrogen on intact and osteotomized bone in osteopenic rats. J Endocrinol 211: 157-168.

Komrakova M, Stuermer EK, Werner C, Wicke M, Kolios L, Sehmisch S, Tezval M, Daub F, Martens T, Witzenhausen P, Dullin C, Stuermer KM (2010) Effect of human parathyroid hormone hPTH (1-34) applied at different regimes on fracture healing and muscle in ovariectomized and healthy rats. Bone 47: 480-492.

Komrakova M, Weidemann A, Dullin C, Ebert J, Tezval M, Stuermer KM, Sehmisch S (2015) The impact of strontium ranelate on metaphyseal bone healing in ovariectomized rats. Calcif Tissue Int 97: 391-401.

Li WL, Yu X, Huang ZP, Pang QJ (2017) Effect of parathyroid hormone on healing in osteoporotic fractures via a phospholipase C-independent pathway. J Int Med Res 45: 1200-1207.

Lips KS, Kauschke V, Hartmann S, Thormann U, Ray S, Kampschulte M, Langheinrich A, Schumacher M, Gelinsky M, Heinemann S, Hanke T, Kautz AR, Schnabelrauch M, Schnettler R, Heiss C, Alt V, Kilian O (2013) Podoplanin immunopositive lymphatic vessels at the implant interface in a rat model of osteoporotic fractures. PLoS One 8: e77259. doi: 10.1371/journal.pone.0077259

Lips KS, Kauschke V, Hartmann S, Thormann U, Ray S, Schumacher M, Gelinsky M, Heinemann S, Hanke T, Kautz AR, Schnabelrauch M, Szalay G, Heiss C, Schnettler R, Alt V, Kilian O (2014) Cholinergic nerve fibers in bone defects of a rat osteoporosis model and their regulation by implantation of bone substitution materials. J Musculoskelet Neuronal Interact 14: 173-188.

Mackert GA, Schulte M, Hirche C, Kotsougiani D, Vogelpohl J, Hoener B, Fiebig T, Kirschner S, Brockmann MA, Lehnhardt M, Kneser U, Harhaus L (2017) Low-energy extracorporeal shockwave therapy (ESWT) improves metaphyseal fracture 
healing in an osteoporotic rat model. PLoS One 12: e0189356. doi: 10.1371/journal.pone.0189356

Marmor M, Alt V, Latta L, Lane J, Rebolledo B, Egol KA, Miclau T (2015) Osteoporotic fracture care: are we closer to gold standards? J Orthop Trauma 29 Suppl 12: S53-56.

Martinez ME, del Campo MT, Medina S, Sanchez M, Sanchez-Cabezudo MJ, Esbrit P, Martinez P, Moreno I, Rodrigo A, Garces MV, Munuera L (1999) Influence of skeletal site of origin and donor age on osteoblastic cell growth and differentiation. Calcif Tissue Int 64: 280-286.

Matos MA, Araujo FP, Paixao FB (2007) The effect of zoledronate on bone remodeling during the healing process. Acta Cir Bras 22: 115-119.

McKibbin B (1978) The biology of fracture healing in long bones. J Bone Joint Surg Br 60-B: 150-162.

Meunier A, Aspenberg P (2006) Parecoxib impairs early metaphyseal bone healing in rats. Arch Orthop Trauma Surg 126: 433-436.

Morgan EF, Mason ZD, Bishop G, Davis AD, Wigner NA, Gerstenfeld LC, Einhorn TA (2008) Combined effects of recombinant human BMP7 (rhBMP-7) and parathyroid hormone (1-34) in metaphyseal bone healing. Bone 43: 1031-1038.

Morse A, McDonald MM, Mikulec K, Schindeler A, Munns CF, Little DG (2020) Pretreatment with pamidronate decreases bone formation but increases callus bone volume in a rat closed fracture model. Calcif Tissue Int 106: 172-179.

Nikolaou VS, Efstathopoulos N, Kontakis G, Kanakaris NK, Giannoudis PV (2009) The influence of osteoporosis in femoral fracture healing time. Injury 40: 663-668.

Nishizuka T, Kurahashi T, Hara T, Hirata H, Kasuga T (2014) Novel intramedullary-fixation technique for long bone fragility fractures using bioresorbable materials. PLoS One 9: e104603. doi: 10.1371/journal.pone.0104603

Nozaka K, Miyakoshi N, Kasukawa Y, Maekawa S, Noguchi H, Shimada Y (2008) Intermittent administration of human parathyroid hormone enhances bone formation and union at the site of cancellous bone osteotomy in normal and ovariectomized rats. Bone 42: 90-97.

Oheim R, Beil FT, Kohne T, Wehner T, Barvencik F, Ignatius A, Amling M, Clarke IJ, Pogoda P (2013) Sheep model for osteoporosis: sustainability and biomechanical relevance of low turnover osteoporosis induced by hypothalamic-pituitary disconnection. J Orthop Res 31: 1067-1074.

Oheim R, Schinke T, Amling M, Pogoda P (2016) Can we induce osteoporosis in animals comparable to the human situation? Injury 47 Suppl 1: S3-9.

Oheim R, Simon MJK, Steiner M, Vettorazzi E, Barvencik F, Ignatius A, Amling M, Clarke IJ, Pogoda P, Beil FT (2017) Sheep model for osteoporosis: The effects of peripheral hormone therapy on centrally induced systemic bone loss in an osteoporotic sheep model. Injury 48: 841-848.
Peng Z, Tuukkanen J, Zhang H, Jamsa T, Vaananen HK (1994) The mechanical strength of bone in different rat models of experimental osteoporosis. Bone 15: 523-532.

Ray S, Thormann U, Eichelroth M, Budak M, Biehl C, Rupp M, Sommer U, El Khassawna T, Alagboso FI, Kampschulte M, Rohnke M, Henss A, Peppler K, Linke V, Quadbeck P, Voigt A, Stenger F, Karl D, Schnettler R, Heiss C, Lips KS, Alt V (2018) Strontium and bisphosphonate coated iron foam scaffolds for osteoporotic fracture defect healing. Biomaterials 157: $1-16$.

Ray S, Thormann U, Sommer U, Khassawna TE, Hundgeburth M, Henss A, Rohnke M, Lips KS, Heiss C, Heinemann S, Hanke T, Durselen L, Schnettler R, Alt V (2016) Effects of macroporous, strontium loaded xerogel-scaffolds on new bone formation in criticalsize metaphyseal fracture defects in ovariectomized rats. Injury 47 Suppl 1: S52-61.

Sandberg O, Aspenberg P (2015a) Different effects of indomethacin on healing of shaft and metaphyseal fractures. Acta Orthop 86: 243-247.

Sandberg O, Aspenberg P (2015b) Glucocorticoids inhibit shaft fracture healing but not metaphyseal bone regeneration under stable mechanical conditions. Bone Joint Res 4: 170-175.

Sandberg O, Aspenberg P (2016) Inter-trabecular bone formation: a specific mechanism for healing of cancellous bone. Acta Orthop 87: 459-465.

Sandberg O, Bernhardsson M, Aspenberg P (2017) Earlier effect of alendronate in mouse metaphyseal versus diaphyseal bone healing. J Orthop Res 35: 793799.

Sandberg O, Eliasson P, Andersson T, Agholme F, Aspenberg P (2012) Etanercept does not impair healing in rat models of tendon or metaphyseal bone injury. Acta Orthop 83: 305-310.

Sandberg O, Macias BR, Aspenberg P (2014) Low dose PTH improves metaphyseal bone healing more when muscles are paralyzed. Bone 63: 15-19.

Saul D, Ninkovic M, Komrakova M, Wolff L, Simka P, Gasimov T, Menger B, Hoffmann DB, Rohde V, Sehmisch S (2018) Effect of zileuton on osteoporotic bone and its healing, expression of bone, and brain genes in rats. J Appl Physiol (1985) 124: 118-130.

Stuermer EK, Komrakova M, Sehmisch S, Tezval M, Dullin C, Schaefer N, Hallecker J, Stuermer KM (2014) Whole body vibration during fracture healing intensifies the effects of estradiol and raloxifene in estrogen-deficient rats. Bone 64: 187-194.

Stuermer EK, Komrakova M, Werner C, Wicke M, Kolios L, Sehmisch S, Tezval M, Utesch C, Mangal O, Zimmer S, Dullin C, Stuermer KM (2010a) Musculoskeletal response to whole-body vibration during fracture healing in intact and ovariectomized rats. Calcif Tissue Int 87: 168-180.

Stuermer EK, Sehmisch S, Rack T, Wenda E, Seidlova-Wuttke D, Tezval M, Wuttke W, Frosch KH, Stuermer KM (2010b) Estrogen and raloxifene improve metaphyseal fracture healing in the early 
phase of osteoporosis. A new fracture-healing model at the tibia in rat. Langenbecks Arch Surg 395: 163172.

Tatting L, Sandberg O, Bernhardsson M, Ernerudh J, Aspenberg P (2017) Isolated metaphyseal injury influences unrelated bones. Acta Orthop 88: 223-230.

Tatting L, Sandberg O, Bernhardsson M, Ernerudh J, Aspenberg P (2018) Different composition of leucocytes in cortical and cancellous bone healing in a mouse model. Bone Joint Res 7: 620-628.

Thormann U, El Khawassna T, Ray S, Duerselen L, Kampschulte M, Lips K, von Dewitz H, Heinemann S, Heiss C, Szalay G, Langheinrich AC, Ignatius A, Schnettler R, Alt V (2014) Differences of bone healing in metaphyseal defect fractures between osteoporotic and physiological bone in rats. Injury 45: 487-493.

Thormann U, Ray S, Sommer U, Elkhassawna T, Rehling T, Hundgeburth M, Henss A, Rohnke M, Janek J, Lips KS, Heiss C, Schlewitz G, Szalay G, Schumacher M, Gelinsky M, Schnettler R, Alt $\mathrm{V}$ (2013) Bone formation induced by strontium modified calcium phosphate cement in critical-size metaphyseal fracture defects in ovariectomized rats. Biomaterials 34: 8589-8598.

Tsiridis E, Morgan EF, Bancroft JM, Song M, Kain M, Gerstenfeld L, Einhorn TA, Bouxsein ML, Tornetta P, 3rd (2007) Effects of OP-1 and PTH in a new experimental model for the study of metaphyseal bone healing. J Orthop Res 25: 1193-1203.

Tu J, Henneicke H, Zhang Y, Stoner S, Cheng TL, Schindeler A, Chen D, Tuckermann J, Cooper MS, Seibel MJ, Zhou H (2014) Disruption of glucocorticoid signaling in chondrocytes delays metaphyseal fracture healing but does not affect normal cartilage and bone development. Bone 69: 12-22.

Uhthoff HK, Goto S, Cerckel PH (1987) Influence of stable fixation on trabecular bone healing: a morphologic assessment in dogs. J Orthop Res 5: 1422.

Uhthoff HK, Rahn BA (1981) Healing patterns of metaphyseal fractures. Clin Orthop Relat Res: 295303.

Wong RM, Thormann U, Choy MH, Chim YN, Li MC, Wang JY, Leung KS, Cheng JC, Alt V, Chow SK, Cheung WH (2019) A metaphyseal fracture rat model for mechanistic studies of osteoporotic bone healing. Eur Cell Mater 37: 420-430.

\section{Discussion with Reviewers}

Stephan Zeiter: One of the important outcomes of this literature review was the finding that many metaphyseal studies heal by endochondral repair rather than only intramembranous ossification. Please discuss the clinical significance of this finding and suggest some key preclinical questions that follow this finding.

Authors: Because osteoporotic fractures frequently occur in metaphyseal bone, it would be highly interesting and clinically relevant to also investigate differences between non-osteoporotic and osteoporotic metaphyseal fracture healing in terms of mechanobiology. One key question would be whether the threshold of mechanical stimuli leading to either endochondral or intramembranous fracture healing might differ dependent on the osteoporosis status of the patients. It has been postulated previously that mechanotransduction in the aged and/or osteoporotic organism differs from young patients. However, this question, to date, is clearly not answered and would be an important subject for further studies.

Stephan Zeiter: The finding that the inflammatory state is different between metaphyseal and diaphyseal fractures is very interesting. Can the authors postulate what the fundamental difference may be and some future studies that could explore this?

Authors: There are very few studies investigating the difference in the inflammatory response occurring after metaphyseal and diaphyseal fractures. These studies postulated that metaphyseal fracture healing displays a rather acute inflammatory response profile, whereas diaphyseal fracture healing displays are more chronic inflammatory profile. However, because the models used in these studies vary greatly in trauma severity, it would be highly pertinent to further analyse this important question in a more standardised setting. Furthermore, it would be highly relevant to shed light on the time course of inflammation between the different types of fractures also regarding different inflammatory cells and molecules.

Chelsea Bahney: This is a great paper and excellent overview. I would challenge the overall concept of it. Why do we need all these imperfect animal models when we have millions of humans suffering these fractures and we are operating on them? We need to be smarter to understand this problem clinically. We have advanced non-radiation involving imaging, we can standardise treatment we can take biopsies at the surgery and during healing, we can take blood tests etc...

Authors: The reviewer is completely correct in that we need more translational and clinical research on the topic of metaphyseal fracture healing. Using advanced clinical imaging techniques would help to understand in which clinical settings metaphyseal fractures heal by endochondral or intramembranous ossification. Taking biopsies, analysing the fracture haematoma, which is frequently removed anyway, and taking blood samples will help to obtain greater insight into biological and molecular processes involved in metaphyseal fracture healing. However, preclinical studies using animal models are absolutely necessary to investigate fracture healing under standardised experimental conditions. In the clinical setting, patient-specific factors like age, sex, drug intake, BMI and many others not currently known might influence the healing in a way we cannot yet predict. Furthermore, there are specific 
research questions which cannot be answered by clinical research, for example, which role does a specific molecule play during bone regeneration and can we utilise this molecule as a target for biological therapy? In conclusion, in an ideal world, preclinical, translational and clinical researchers would work together on important orthopaedic topics with the goal of benefitting the patient.

Zsolt Balogh: A very important aspect of preclnical studies is their reproducibility. The insufficient/ inappropriate reporting of animal studies has been discussed extensively (e.g. ARRIVE guidelines) as a possible reason for difficulties in reproducing published work. Based on the papers included in this review, do the authors think that these models have been described in sufficient detail to be able reproduce them? I would especially be interested in the description of the surgical technique. This could be an interesting aspect to be included in the manuscript.

Authors: The reviewer raised a very important point. Indeed, many of the published studies on metaphyseal fracture healing reported about adverse effects, animal welfare aspects and inclusion/ exclusion criteria for animals as it is recommended by the ARRIVE guidelines (although many papers were published before these guidelines were established). However, not all studies included these data, and the description of the surgical technique is frequently not detailed enough to allow repetition of the experiment. We added this information to the summary statement of the manuscript.

Editor's note: The Scientific Editor responsible for this paper was Chris Evans. 